Research Paper:

\title{
Effects of Chronic Administration of Pioglitazone on Learning and Memory in Rat Model of Streptozotocin-induced Alzheimer's Disease
}

\author{
Ehsan Aali ${ }^{1}$, ${ }^{*}$ Mohammad Hossein Esmaeili $^{2}$ (C), Sead Shima Mahmodi ${ }^{1}{ }^{\circ}$, Poriea Solimani ${ }^{1}$
}

1. Department of Pharmacology, School of Medicine, Qazvin University of Medical Sciences, Qazvin, Iran.

2. Department of Physiology, School of Medicine, Qazvin University of Medical Sciences, Qazvin, Iran.

\begin{tabular}{|c|c|}
\hline $\begin{array}{l}\text { Use your device to scan } \\
\text { and read the article online }\end{array}$ & Citation Aali E, Esmaeili MH, Mahmodi SSh, Solimani P. Effects of Chronic Administration of Pioglitazone on Learning and Memory \\
\hline Pyprin & $\begin{array}{l}\text { in Rat Model of Streptozotocin-induced Alzheimer's Disease. Journal of Inflammatory Diseases. 2020; 24(4):294-307. https://doi. } \\
\text { org/10.32598/JQUMS.24.4.1 }\end{array}$ \\
\hline 문단 & doi'https://doi.org/10.32598/JQUMS.24.4.1 \\
\hline
\end{tabular}

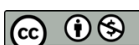

Received: 21 Apr 2020

Accepted: 14 Sep 2020

Available Online: 01 Oct 2020

Keywords:

Pioglitazone, Memory,

Insulin resistance,

Streptozotocin

\section{A B STRACT}

Background Alzheimer's Disease (AD) is a chronic neurodegenerative disease characterized by abnormal protein accumulation, synaptic dysfunction, and cognitive impairment. Peroxisome Proliferator-Activated Receptor- $\gamma$ (PPAR $\gamma$ ) play a crucial role in regulating insulin sensitivity and may serve as potential therapeutic targets for AD. Pioglitazone (PIOG), as a PPAR $y$ agonist, reduces $\beta$-amyloid and tau proteins, and inhibits neuroinflammation.

Objective This study aims to evaluate the effects of PIOG chronic administration on learning and memory in rat model of Streptozotocin (STZ)-induced AD

Methods Forty-two male Wistar rats were divided into two groups: A. Normal rats divided into three subgroups of Control, Dimethyl Sulfoxide (DMSO), and PIOG; and B. AD rats divided into four subgroups of Vehicle, STZ, STZ+DMSO and STZ+PIOG. The last two AD subgroups received $0.2 \mathrm{~mL}$ DMSO and PIOG (10 $\mathrm{mg} / \mathrm{kg}$ per day) for 21 days. For induction of $A D, S T Z$ ( $3 \mathrm{mg} / \mathrm{kg}, 10 \mu$ per injection site) were administered into lateral ventricles. All rates were trained under the Morris water maze task.

Findings PIOG impaired the spatial learning and memory in normal rats. Intracerebroventricular injection of STZ significantly increased escape latency and swimming time to find the hidden platform compared to the control group $(\mathrm{P}<0.05)$. The amnesic effect of STZ was prevented by PIOG administration such that the escape latency and swimming time to find the hidden platform in the STZ+PIOG group were significantly lower than in the STZ+DMSO group $(\mathrm{P}<0.05)$. Conversely, the percentage of time spent and distance swimming in the target quadrant in the probe test in the STZ+ PIOG group rats were significantly higher than those in the STZ + DMSO group.

Conclusion PIOG administration impaired spatial learning and memory in normal rats, but improved learning and memory in rats with STZ-induced AD. It can be useful for treatment of cognitive impairment in $A D$ patients.

\section{Extended Abstract}

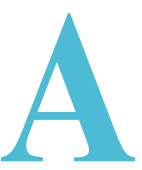

\section{Introduction}

lzheimer's Disease (AD) is the most common neurodegenerative disease character- ized by progressive memory impairment [1]. Most of recent studies have shown an increased risk of $A D$ in people with diabetes [2]. AD is closely associated with impaired insulin signaling in brain, suggesting it to be a brain-specific form of diabetes [3]. Thus, the most important method of reducing memory impairment in

\section{* Corresponding Author:}

Mohammad Hossein Esmaeili

Address: Department of Physiology, School of Medicine, Qazvin University of Medical Sciences, Qazvin, Iran.

Tel:+98 (28) 33236001

E-Mail: mesmaili@qums.ac.ir 
patients with $\mathrm{AD}$ is to target insulin signaling pathway. In this regard, it has been demonstrated that antidiabetic drugs such as metformin can be effective in the treatment of memory impairment in AD people. Peroxisome Proliferator-Activated Receptor- $\gamma$ (PPAR $\gamma$ ) is a transcription factor that regulates numerous genes involved in regulation of cell differentiation and metabolism [24]. PPAR- $\gamma$ agonists (thiazolidinediones) are widely used in clinical medicine for the treatment of type 2 diabetes mellitus. Recently, PPAR- $\gamma$ agonists have shown neuroprotective effects on neurodegenerative diseases. Pioglitazone (PIOG), a PPAR $\gamma$ agonist, has effective insulin-sensitizing, anti-inflammatory, neuroprotective, and anti-excitotoxic properties that make it a good candidate for improving cognition in insulin-resistant individuals. The current study aims to investigate the effects of chronic administration of PIOG on cognitive impairment in rat model of AD.

\section{Materials and Methods}

\section{Samples}

Male Wistar rats (180-200 g) were housed in standard polycarbonate cages. They were given ad libitum access to Food and water. Animals were divided into two groups: Control group received Dimethyl Sulfoxide (DMSO) plus PIOG, and AD group divided into four subgroups of Vehicle (normal saline), STZ, STZ+DMSO and STZ+PIOG. The last two subgroups received DMSO and PIOG (10 mg/kg per day) for 21 days [21].

\section{Induction of AD}

For induction of AD, Streptozotocin (STZ) was administered into lateral ventricles of rats. To do this, rats were first anesthetized with an Intraperitoneal (IP) injection of ketamine $(60 \mathrm{mg} / \mathrm{kg})$ and xylazine $(8 \mathrm{mg} / \mathrm{kg})$ and placed in a Stoelting stereotaxic instrument. STZ (3 $\mathrm{mg} / \mathrm{kg}, 5 \mu \mathrm{l} /$ injection site) was dissolved in normal salin [9]. STZ or vehicle was administered by Intracerebroventricular (ICV) injection into lateral ventricles: Anteroposterior $(\mathrm{AP})=-0.8 \mathrm{~mm}$; Mediolateral $(\mathrm{ML})= \pm 1.5 \mathrm{~mm}$; Dorsoventral $(\mathrm{DV})=-3.6 \mathrm{~mm}$.

\section{Morris Water Maze test}

The Morris water maze test was performed as described in Reference [37]. Escape latency was used to show the learning ability. The time spent in the platform quadrant was recorded to indicate the spatial memory.

\section{Data analysis}

The collected data were analyzed by using one-way ANOVA and Tukey's post hoc test. All data were presented using Standard Error of the Mean (SEM). A $\mathrm{P}<0.05$ was considered as statistically significance level.

\section{Results}

PIOG caused impairment in spatial learning and memory in normal rats (Figure 1). The ICV injection of STZ significantly increased escape latency and swimming time to find the hidden platform compared to the control group $(\mathrm{P}<0.05)$. The amnesic effect of STZ was prevented by treatment with PIOG such that the escape latency and swimming time to find the platform in the STZ+PIOG group were significantly lower than in the STZ+DMSO groups $(\mathrm{P}<0.05)$. Conversely, the escape latency and the time spent in the target quadrant under the probe test in the STZ+PIOG group were significantly higher than in the STZ+DMSO group (Figure 2).

\section{Discussion}

The findings showed that PIOG administration in normal rats impaired their spatial learning and memory but improved the learning and memory in rats with STZinduced AD. STZ caused longer escape latencies in the training trials than the control rat indicating impairment in cognitive function by STZ. The prolongation of escape latency by STZ was significantly and gradually decreased after five testing days by PIOG administration. In addition, PIOG significantly reduced the effect of STZ on swimming time spent to find hidden platform in the target quadrant under the probe test. Therefore, chronic administration of PIOG $(10 \mathrm{mg} / \mathrm{kg}$, IP for 21 days) prevented the STZ-induced deficits in memory and cognition. The results suggest that PIOG is useful for treatment of cognitive impairment in $\mathrm{AD}$ patients.

The improvement of spatial learning and memory by PIOG administration reported in our present study is in line with the results of previous studies. For example, Pathan et al. (2006) demonstrated that oral administration of PIOG improved cognitive function under stepthrough passive avoidance task in diabetic rats. Similar results have been reported by Baraka and ElGhotny (2010), where AD was induced by ICV injection of $\beta$-amyloid in rats and significant improvement in spatial learning and memory was observed after treatment daily by oral PIOG administration for 3 weeks. Consistent with our results, Allami et al. showed that PIOG improved the memory impaired by scopolamine under 

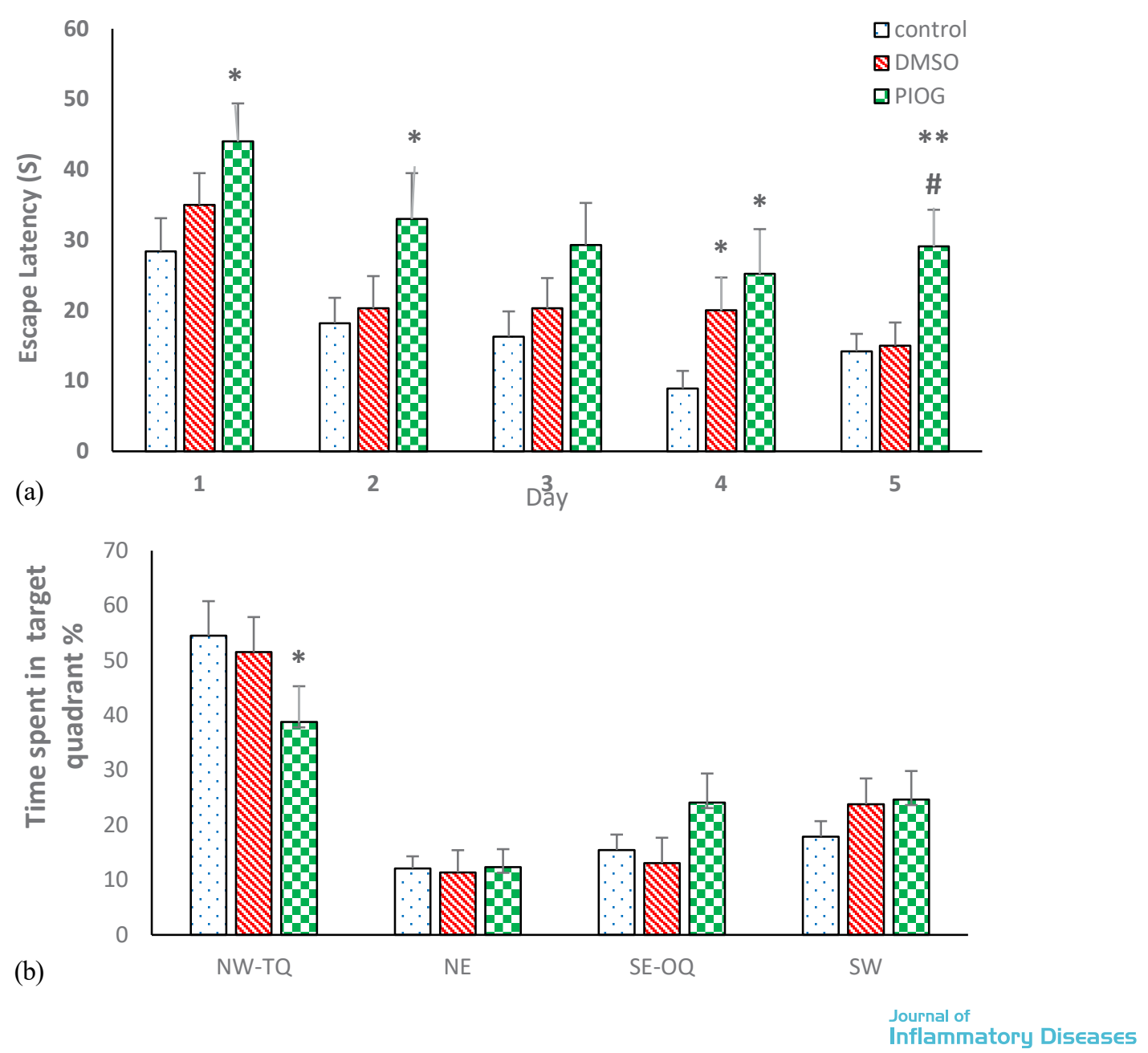

Figure 1. Effects of PIOG administration on spatial learning and memory in normal rats under the Morris Water Maze test

Values are presented as $+\mathrm{SEM}$ of A: Escape latency; and B: Time spent in the target quadrant;

${ }^{*} \mathrm{P}<0.05$; and ${ }^{* *} \mathrm{P}<0.01$ compared to control group; ${ }^{*} \mathrm{P}<0.05$ compared to DMSO group; NW-TQ: North West Target Quadrant; SE-OQ: South East Opposite Quadrant; SW: South West; NE: North East.

Y-maze test, whereas it did not affect scopolamine-induced cognitive impairment under the passive avoidance test. This discrepancy can be related to differences in setting, dosage and duration. In contrast to our study, Nicolakakis et al. (2008) reported that PIOG could not improve memory in transgenic mice model of AD [15]. This discrepancy can also be due to shorter duration of treatment with PIOG and/or the use of transgenic mice with two mutations in their study.

\section{Conclusion}

Chronic administration of PIOG $(10 \mathrm{mg} / \mathrm{kg}$, IP for 21 days) prevents the STZ-induced memory and learning impairments. It can be useful for treatment of cognitive impairment in $\mathrm{AD}$ patients.

\section{Ethical Considerations}

\section{Compliance with ethical guidelines}

This study approved by the Research Ethics Committee of Qazvin University of Medical Sciences (Code: IR.QUMS.REC. 1398.158).

\section{Funding}

This study was extracted from the MD. thesis of third author, Department of Physiology, School of Medicine, 

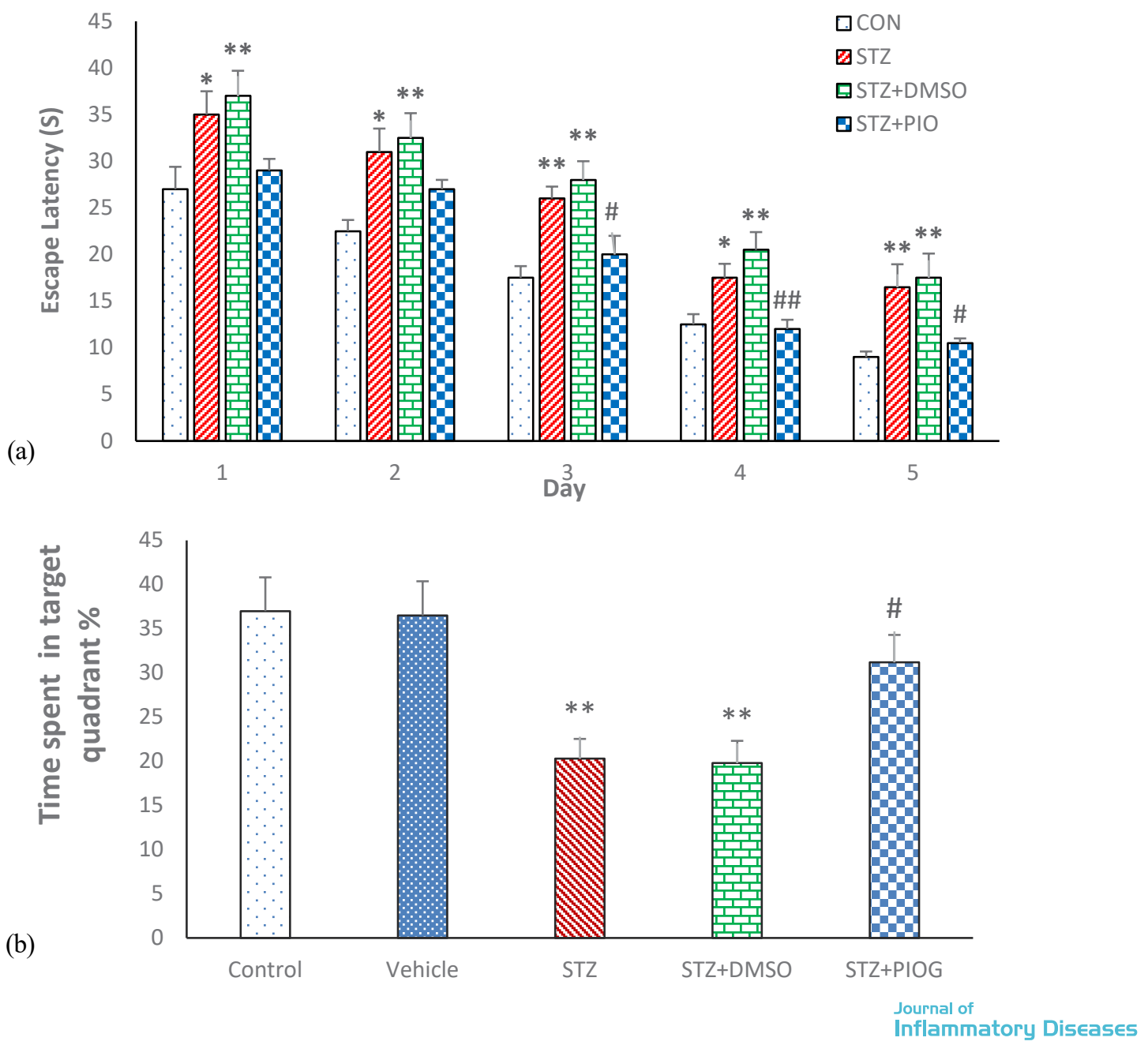

Figure 2. Effects of PIOG administration on spatial learning and memory in rats with sporadic AD under the Morris Water Maze test

Values are presented as+SEM of A: Escape latency; and B: Time spent in the Target Quadrant (TQ);

${ }^{*} \mathrm{P}<0.05$; and ${ }^{* *} \mathrm{P}<0.01$ compared to control group; ${ }^{*} \mathrm{P}<0.05$; and ${ }^{\#} \mathrm{P}<0.01$ compared to STZ+DMSO group.

Qazvin University of Medical Sciences and this study did not receive any specific grant from funding agencies in the public, commercial, or not-for-profit sectors.

\section{Authors' contributions}

Writing: All authors; Resources and validation: Sead Shima Mahmodi and Poriea Solimani; Editing and project administration: Ehsan Aali; Data analysis and methodology: Mohammad Hossein Esmaeili.

\section{Conflicts of interest}

The authors declared no conflict of interest. 
This Page Intentionally Left Blank 


\title{
اثرات تجويز مزمن بيوگكليتازون بر يادتيرى و حافظه فضايى موشهاى سالم و آلزايمرى مدل استريتوزوتوسين
}

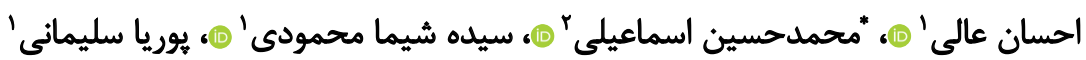

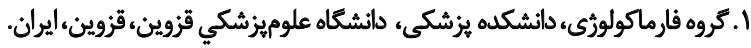

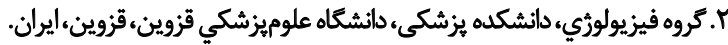

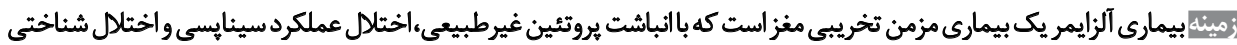

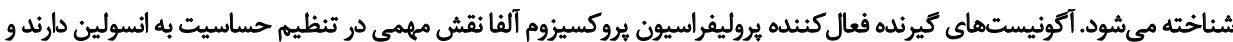

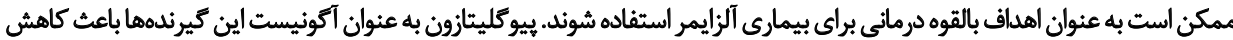

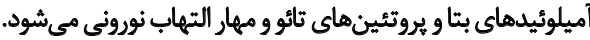

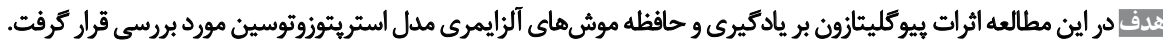

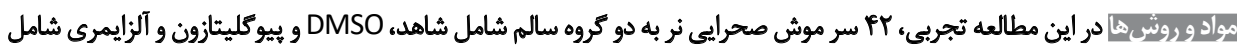

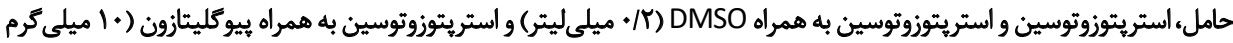

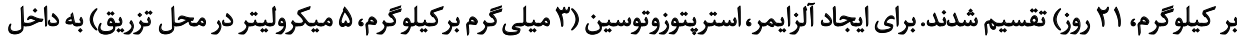

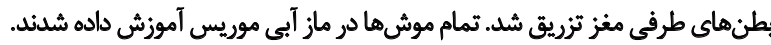

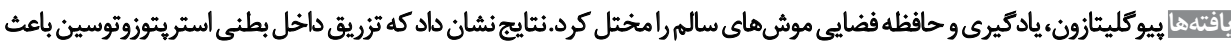

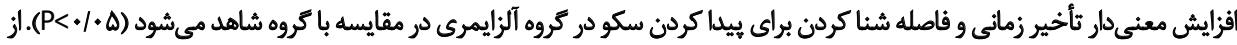

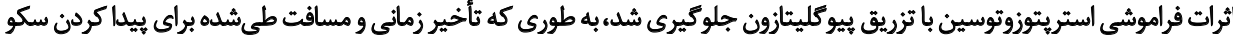

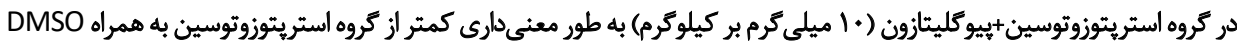

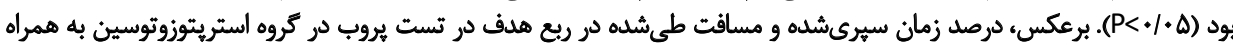

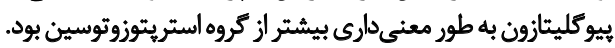

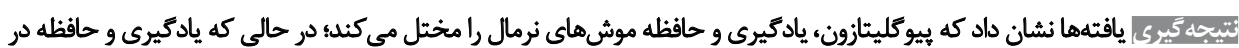

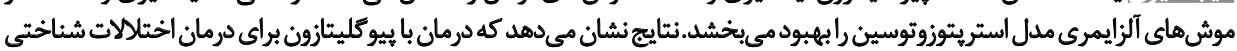
در بيماران آلزايمرى مفيد أستيت.

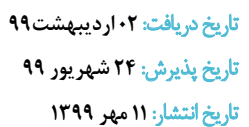

كليدوأوهها: بيو كليتازون، حاقظظه،

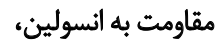
استريتوزوتوسين بن انسين

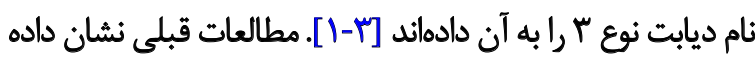

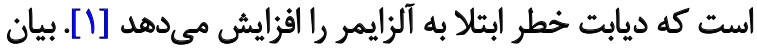

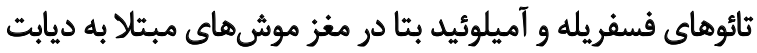

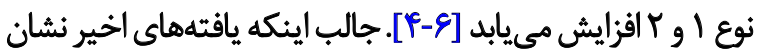

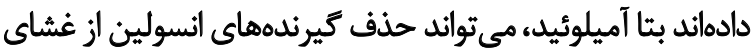

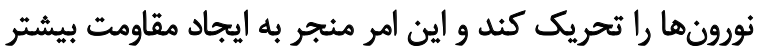

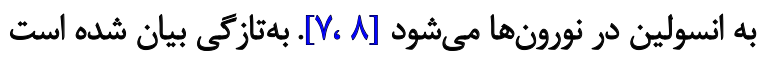

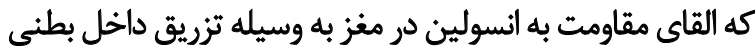

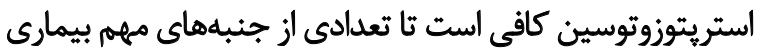

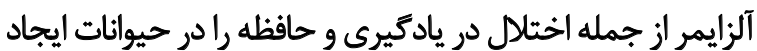

مقاd

تجمع داخل و خارج سلولى آميلوئيد بتا و يروتئينهاى

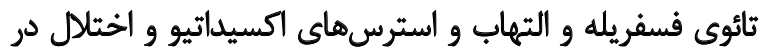

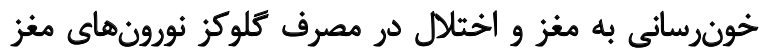

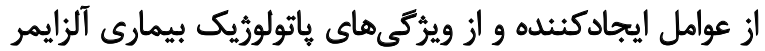

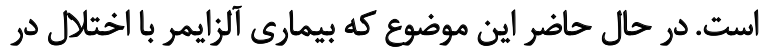

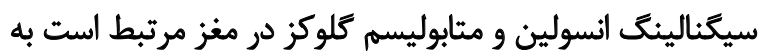

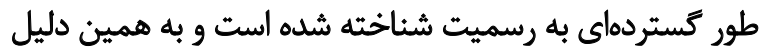

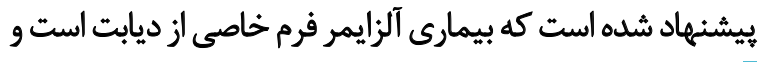

* ثميسنده مسئول:

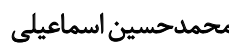

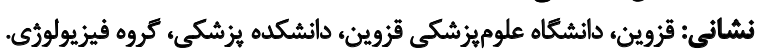

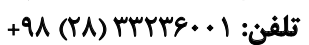
رايانامه: mesmaili@qums.ac.ir 
در مرحله دوم آزمايش، تأثير داروى بيوكَليتازون بر يادكيرى

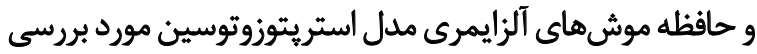

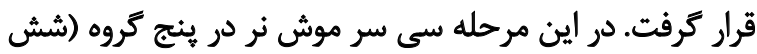

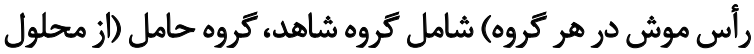

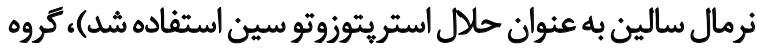

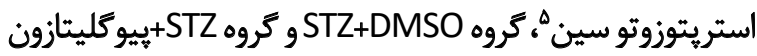

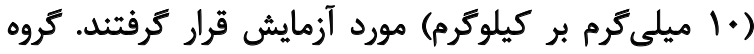
و وروه STZ DSO+STZ

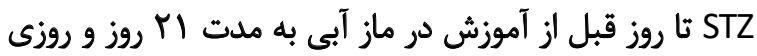

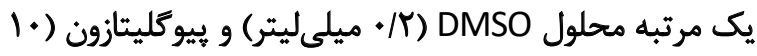
ميلى گرم بر كيلوكرم) به صورت درون صفاقى دريافت كردند.

براى القاى آلزايمر، ه ميكروليتر از محلول استريتوزوتوسين با بإيا

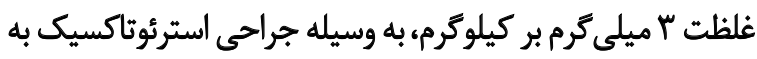

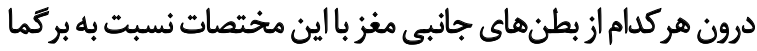

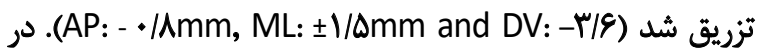

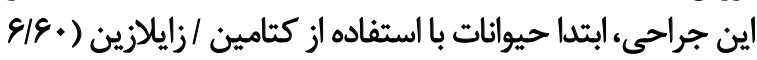

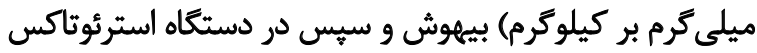

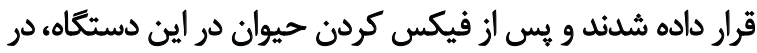

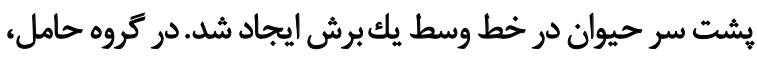

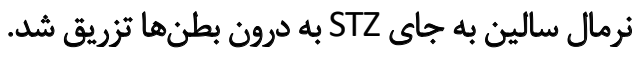

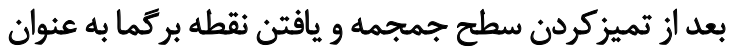

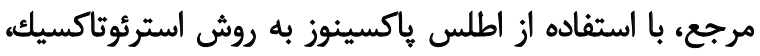

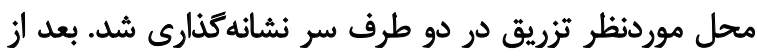

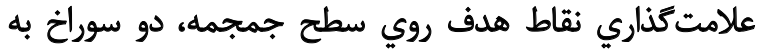

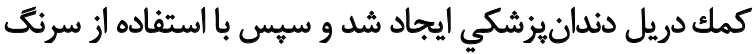

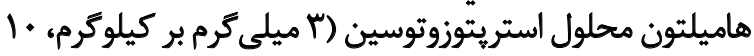

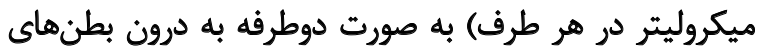
جانبى بهآرامى تزريق شد.

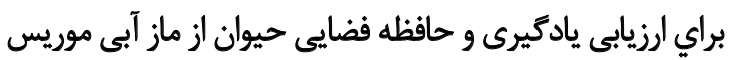

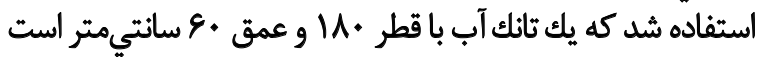

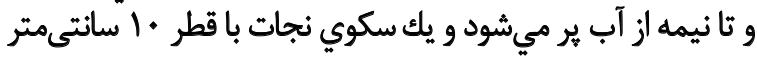

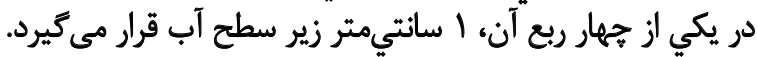

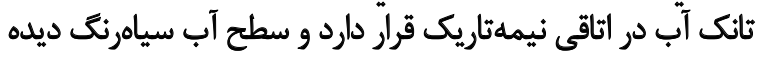

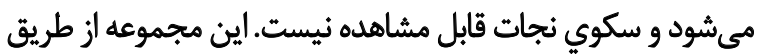

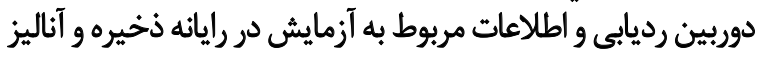

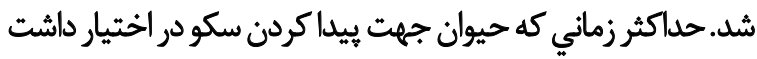

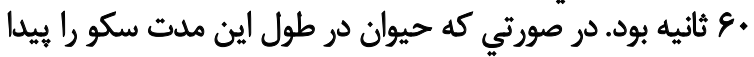

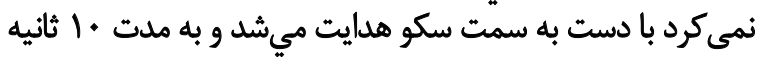

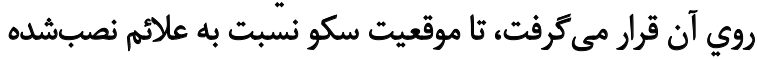

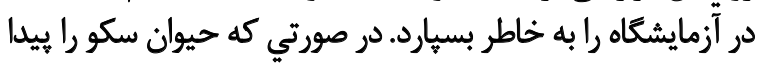

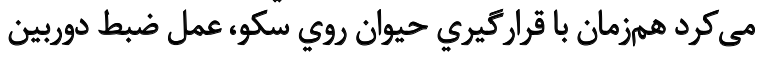

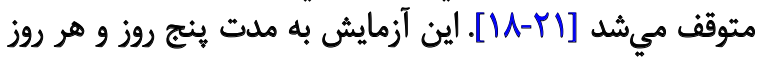

كند [9]. بنابراين بهبود خونرسانى به مغز و كاهش مقاومت

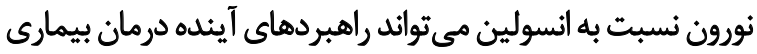

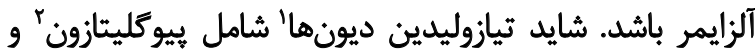

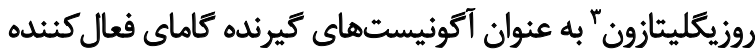

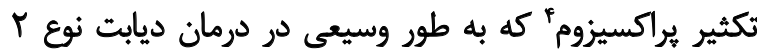

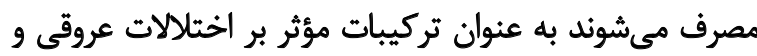

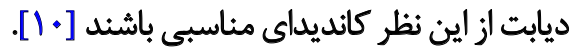

نقش Y PPAR تنظيم بيان رنهايى است كه منجر به تنظيمي

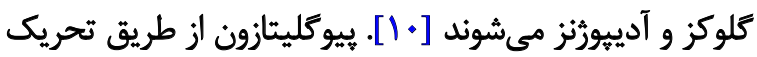

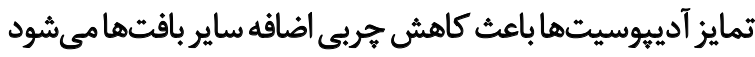

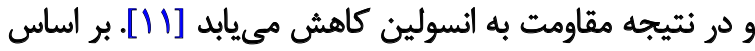

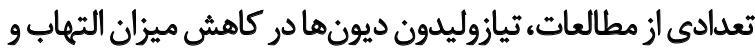

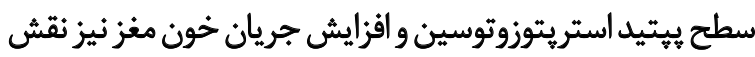

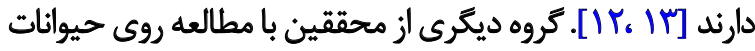

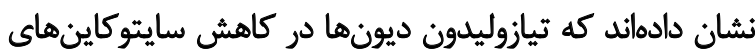

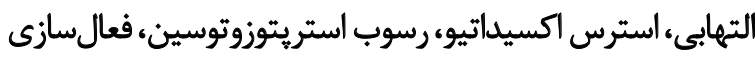

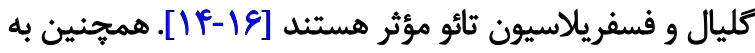

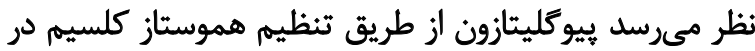

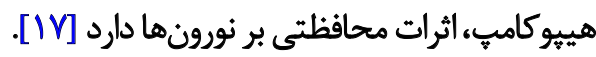

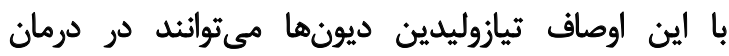

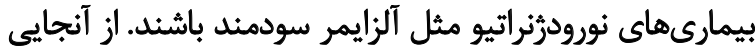

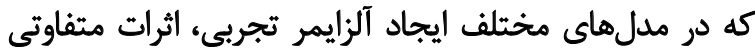

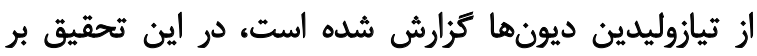

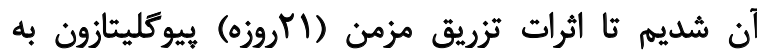

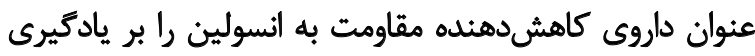

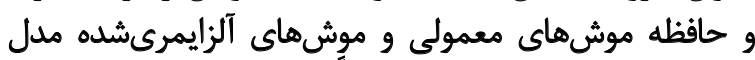

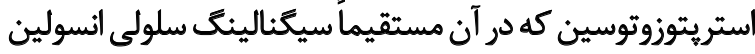

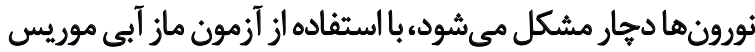

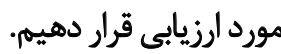

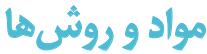

اين مطالعه تجربي، در كروه فيزيولوزى دانشكاه علوميزشكى دري

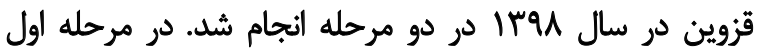

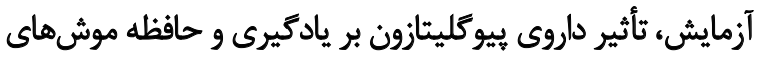

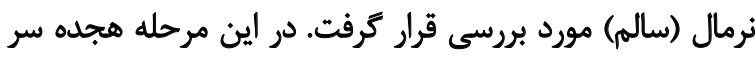

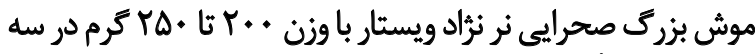

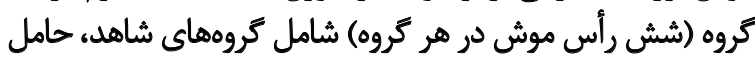

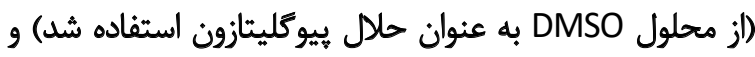

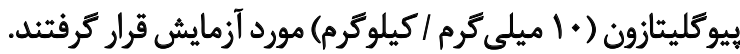

1. Thiazolidinediones

2. Pioglitazone

3. Rosiglitazone

4. Peroxisome Proliferator-Activated Receptor $y$ (PPARs) 
در تجزيه و تحليل دادهها براي بررسي تفاوتهاي درون تروهي

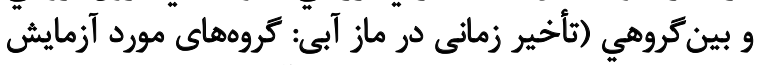

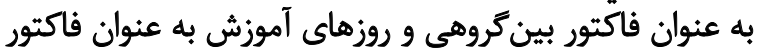

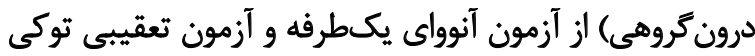

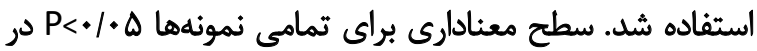

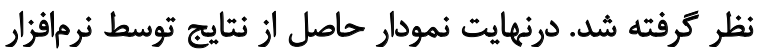
اكسل ترسيم شد.

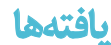

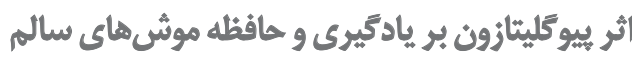
همانطور كه در شكل شماره ا مشاهده مىشود، تزريق مزمن

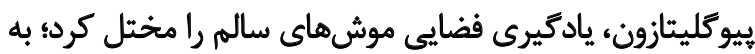

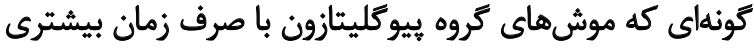

جهار بار با فاصله • ا دقيقه تكرار شد. در اين مراحل روند يادكيري

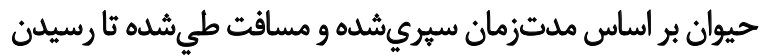

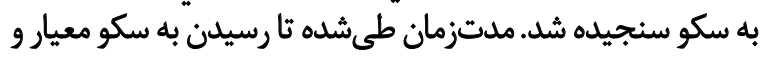
ميزان سنجش يادكيرى بود.

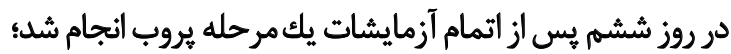

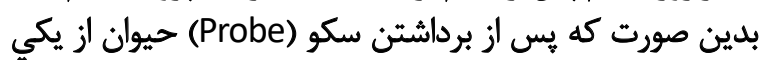

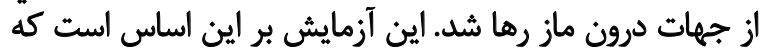

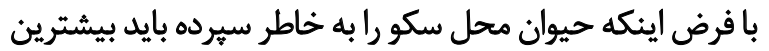

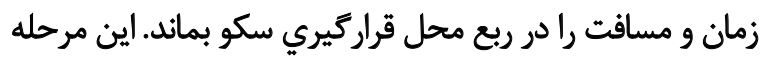

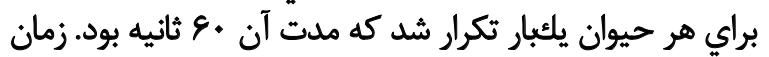

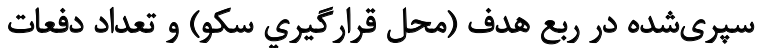

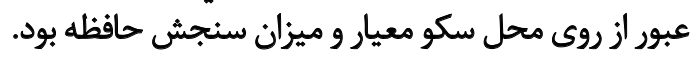
دادههاى حاصل از مطالعه وارد نرمافزرار SPSS نسخه Fr شدند.

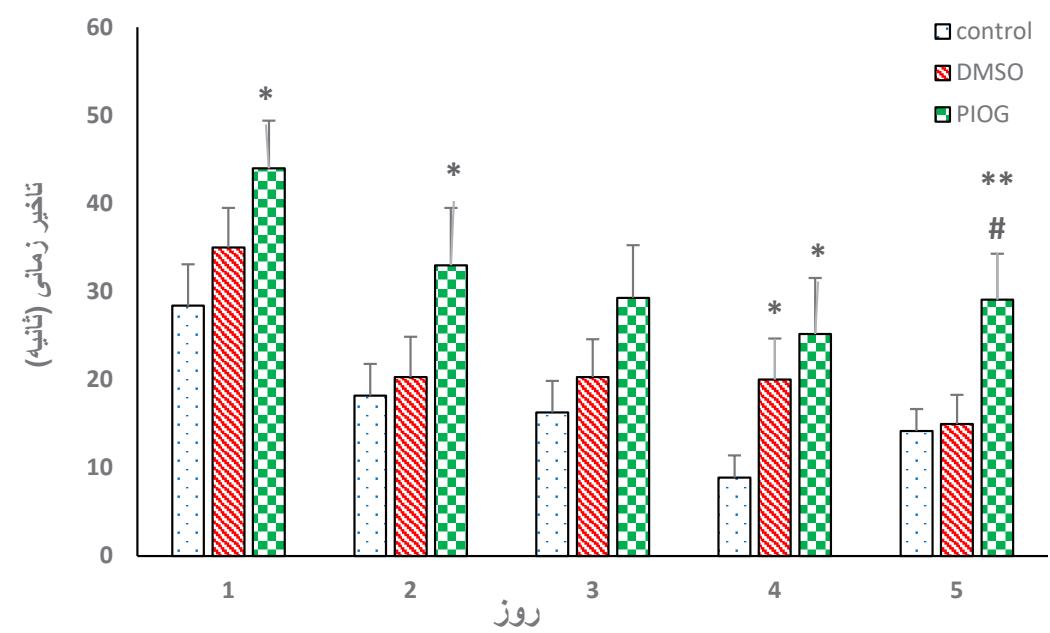

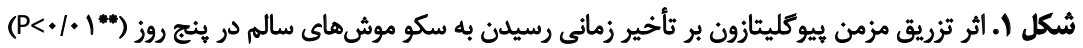

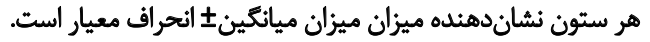

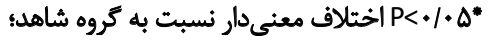

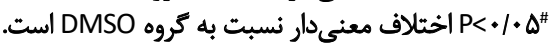

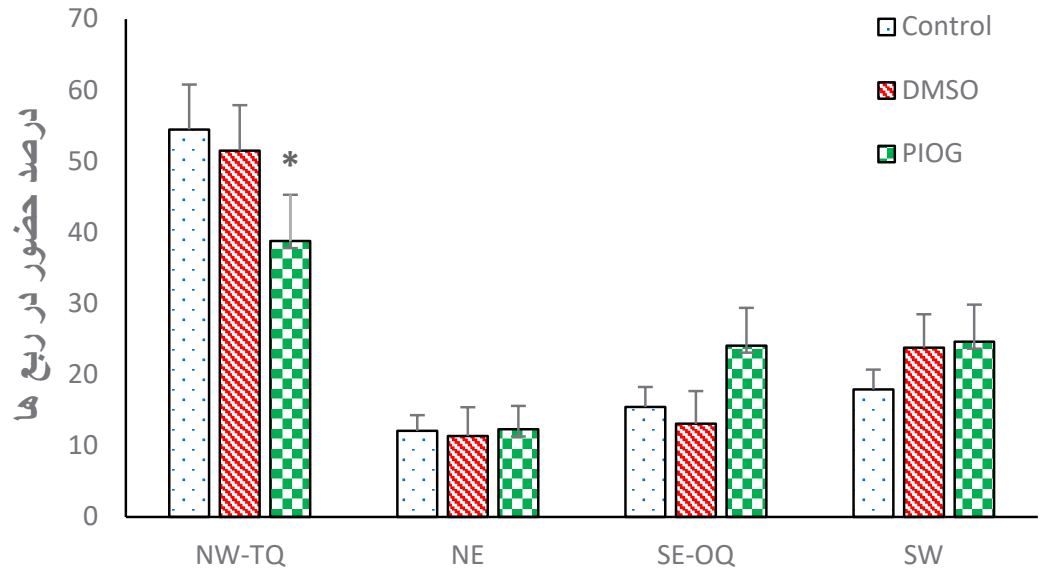

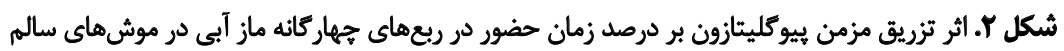

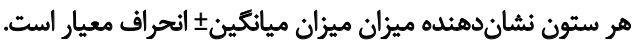
اختلاف معنى دار نسبت به كروه شاهند 


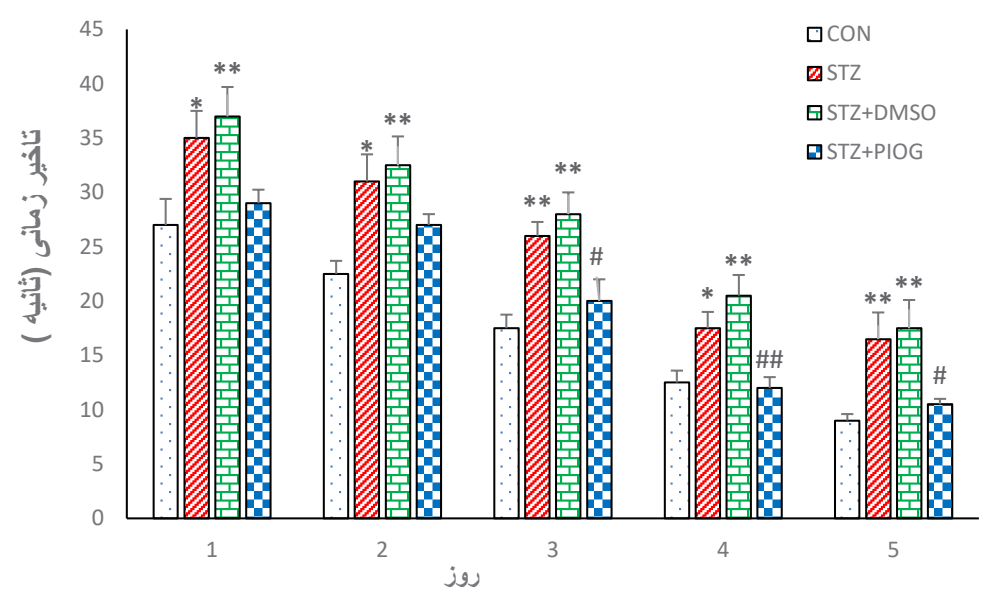

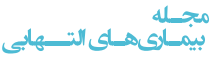

شكل "ب. اثر تزريق مزمن بيوكليتازون (• ميلى كرم بر كيلوكرم، درونصفاقى) بر تأخير زمانى رسيدن به سكو در طول بنج روز آموزش موشهاى آلزايمرى مدل

استريتوزوثوسين

هر ستون نشاندهنده ميزان ميزان ميانكين ثانحراف معيار است.

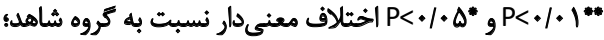
STZ اختلاف معنى دار نسبت به كرون

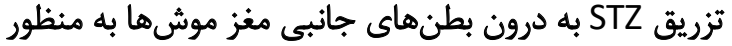

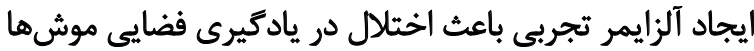

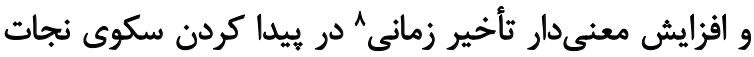

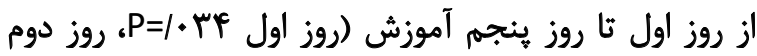

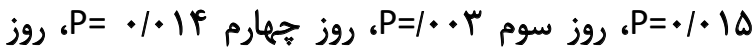

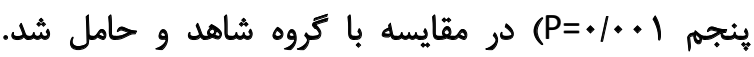

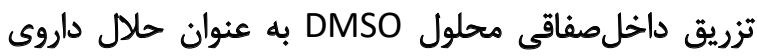

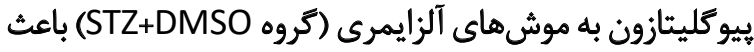

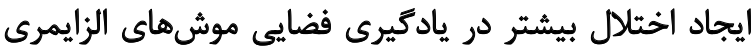

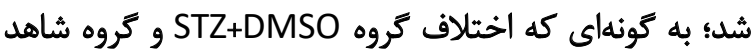

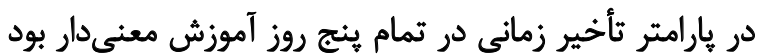
(P<•/・・1)

همجنين نتايج نشان داد كه درمان موشهاى آلزايمرى با بإن

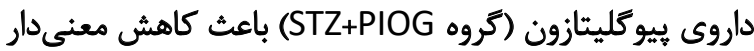

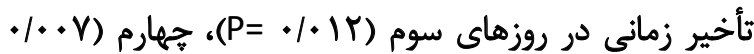

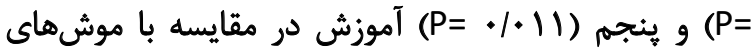

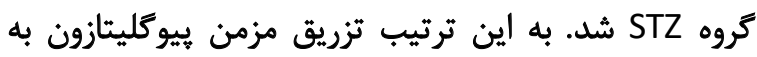

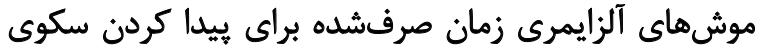

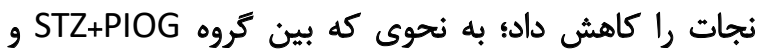

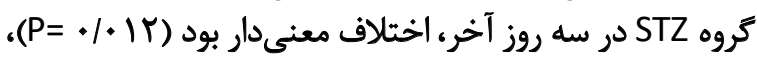

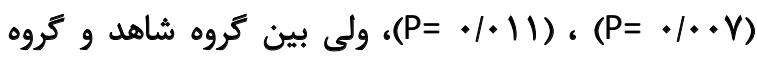
STZ+PIOG

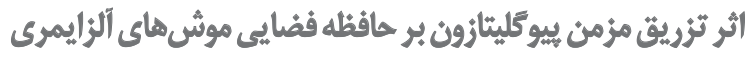
همانطور كه در شكلهاى شماره f و ه نشان داده شده است

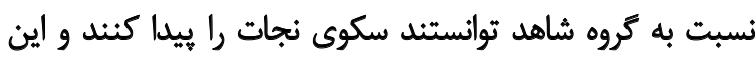

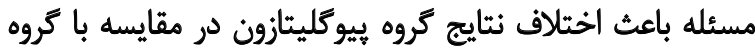

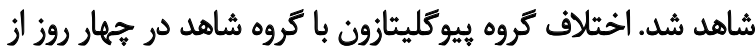

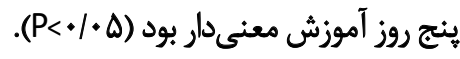

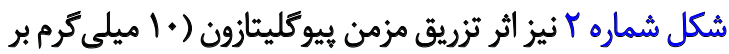

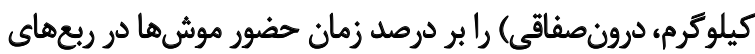

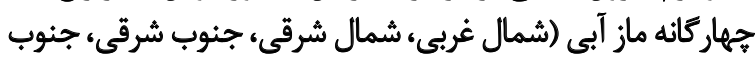

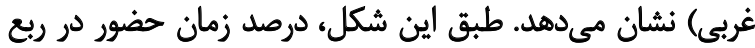

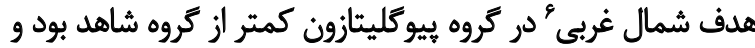

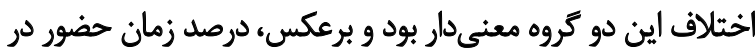

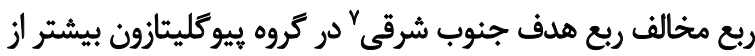

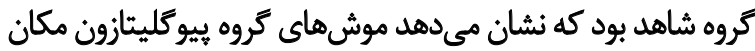

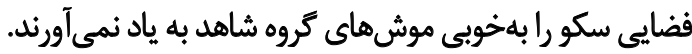

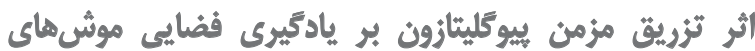
ألزايمرى أنز

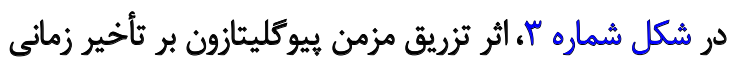

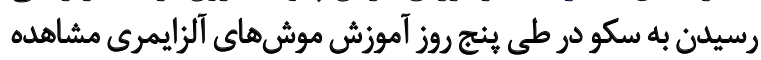

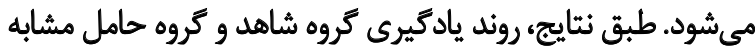

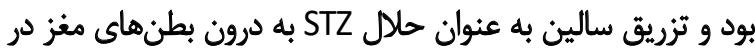

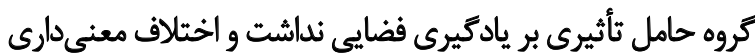

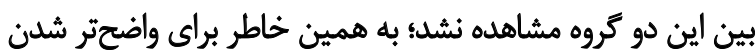

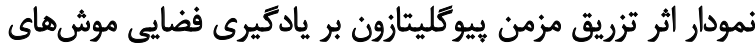

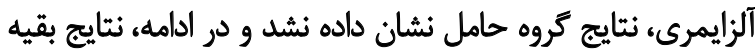

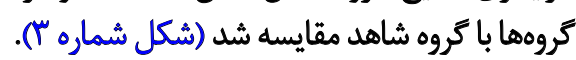




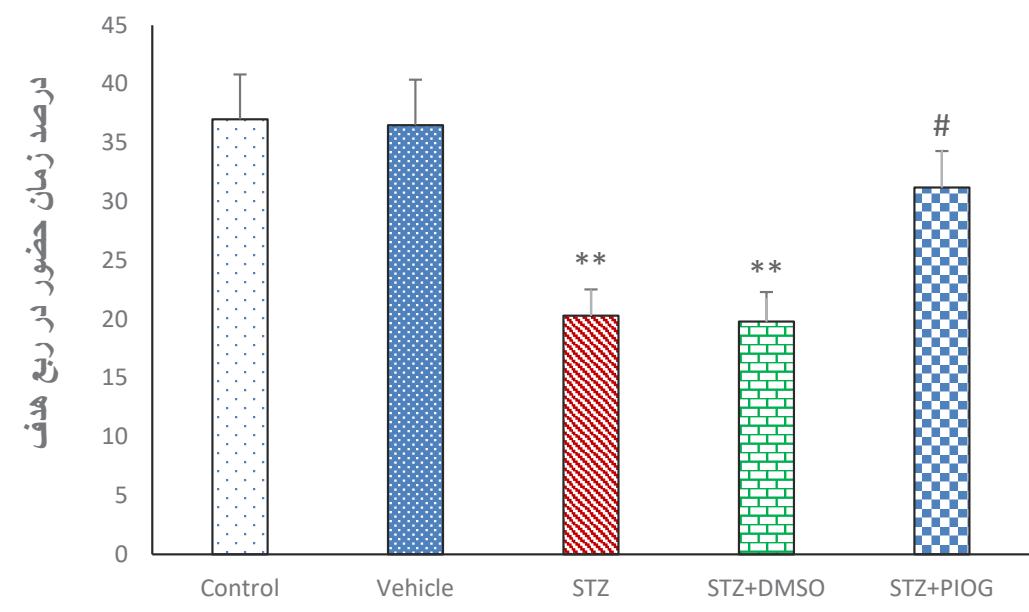

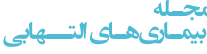

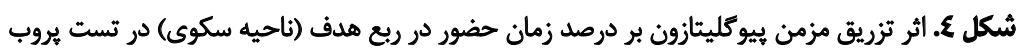

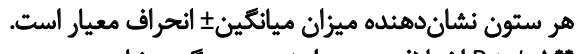

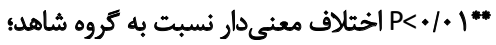

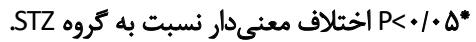

مزمن بيوكليثازون به موشهاي آلزايمرى منجر به افزايش درصد

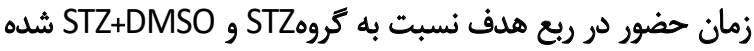

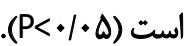

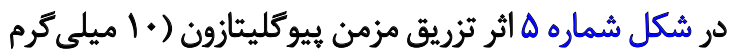

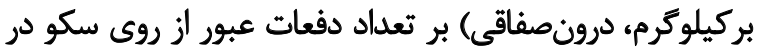

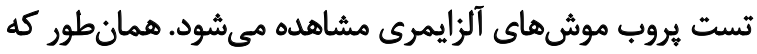

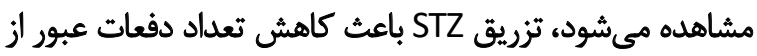

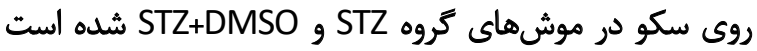

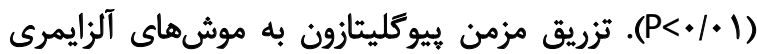

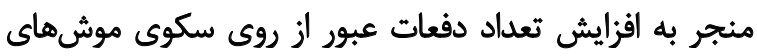
آلزايمرى نسبت به كروه STZ و اين افزايش معنى دار نبود.
تزريق STZ به درون بطنهاى جائبى مغز موشها به منظور ايجاد

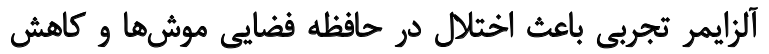
معنى دار زمان صرفشده در ربع هدف سكو و تعداد دفعات عبور

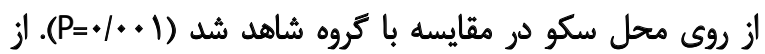

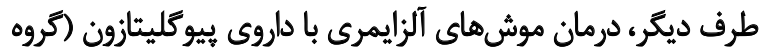

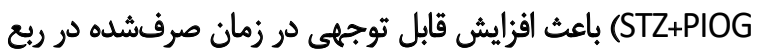

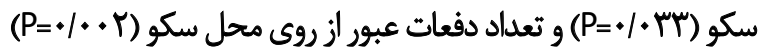

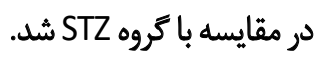

در شكل شماره F اثر تزريق مزمن بيوكليتازون (•1 ميلى مئرم

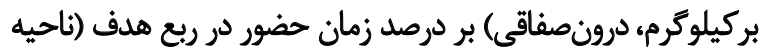

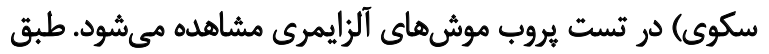

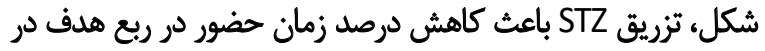

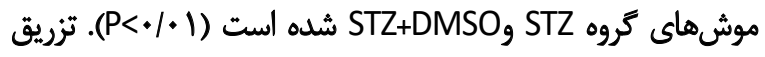

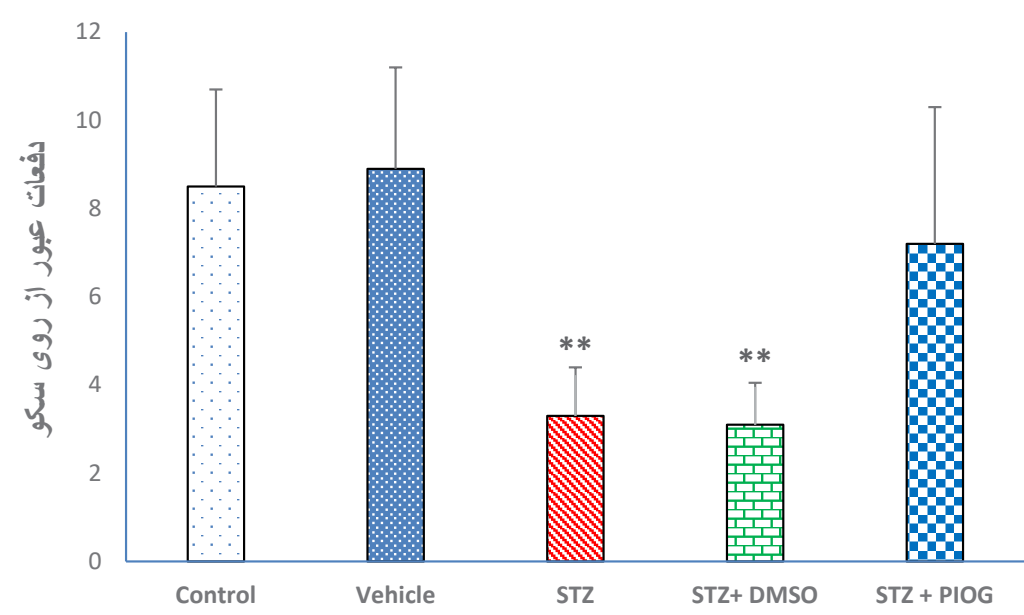

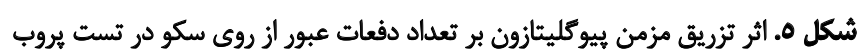

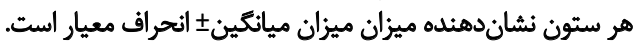

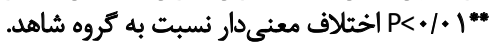


آلزايمر و هاركينسون سودمند باشند. در تأييد اين ويشبيشيني،

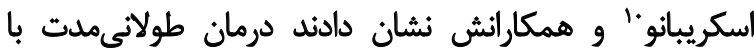

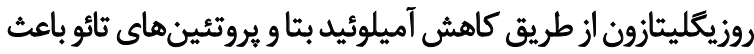

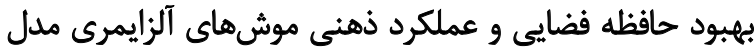

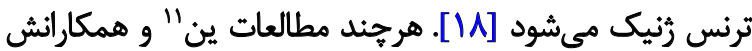

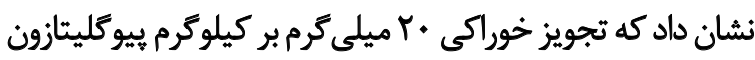

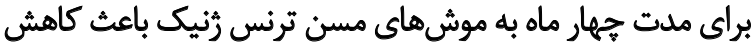

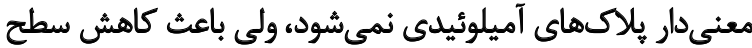

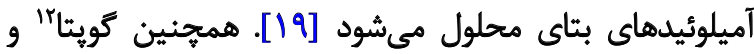

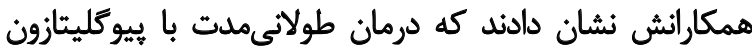

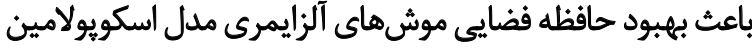

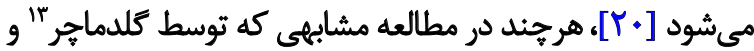

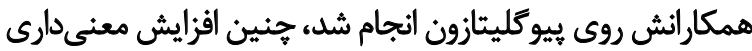

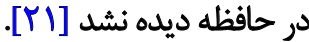
اولين مكانيسم مطرح در اين مورد، بهبود سيكنالينك سلولى

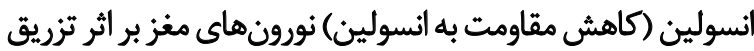

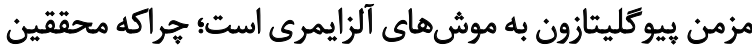

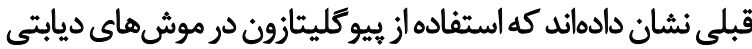

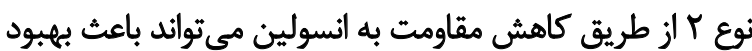

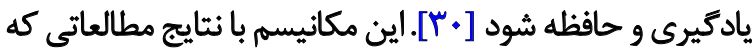

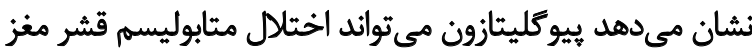

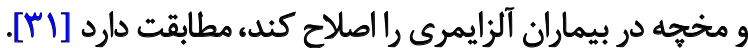

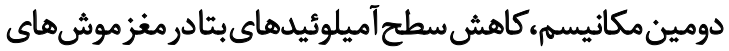

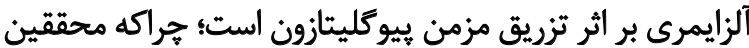

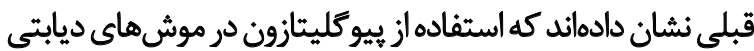

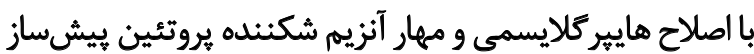

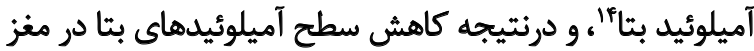

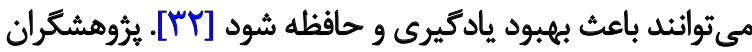

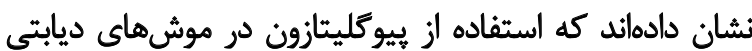

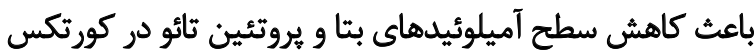

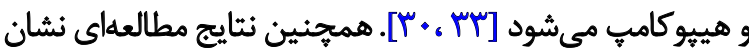

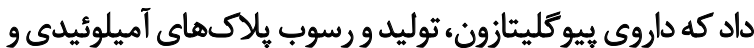

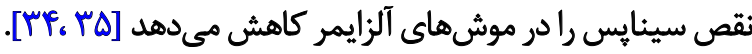
مكانيسم سوم در اين مورد، كاهش ميزان التهاب و كاهش

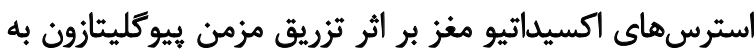

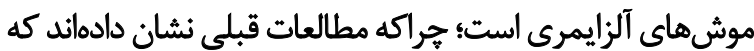

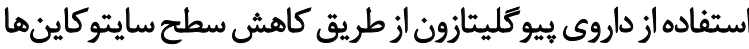

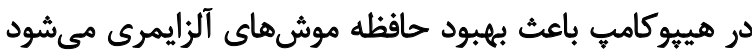

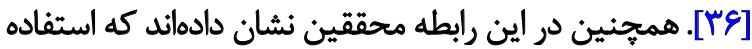

10. Escribano

11. Yan

12. Gupta

13. Geldmacher

14. $\beta$-amyloid precursor protein cleaving enzyme 1 (BACE1)
سرعت شناى موشهاى كروههاى مورد آزمايش نيز مقايسه

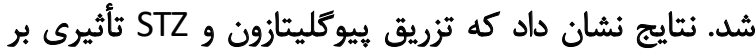

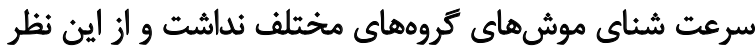

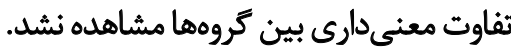

\section{بحث ونتيجهان كيرى}

نتايج مرحله اول مطالعه ما نشان داد كه تزريق مزمين

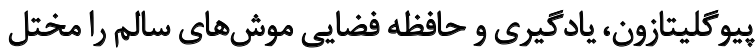

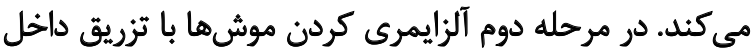

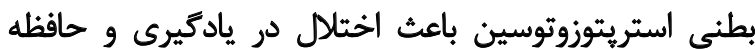

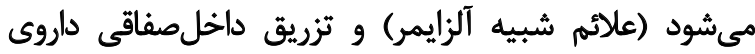

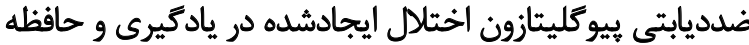

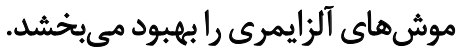
سؤال مهمم اين است كه بيوكليتازون از طريق كدام مكام مكانيسم

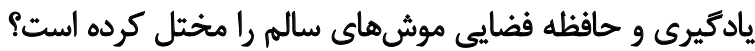

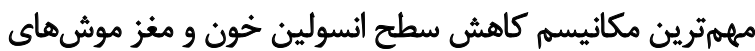

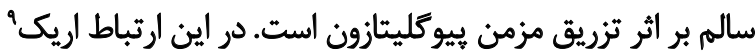

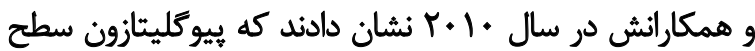

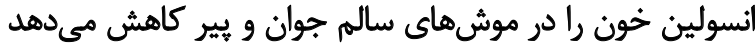

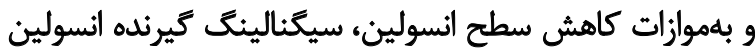

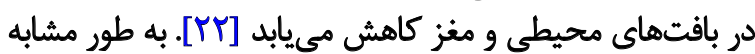

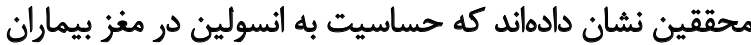

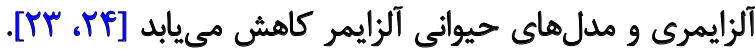

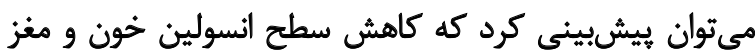
اثرى مشابه كاهش حساسيت مغز نسبت به به انسولين داني در نورونه

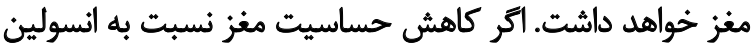

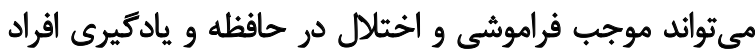

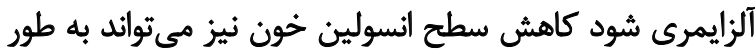

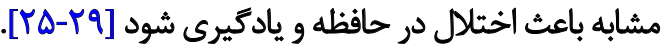
نتايج مرحله دوم مطالعه حاضر نشان داد كه آلزايمرى

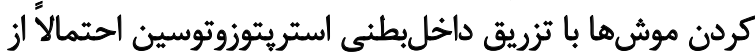

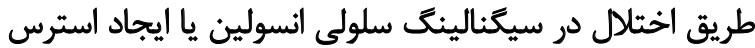

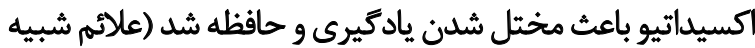

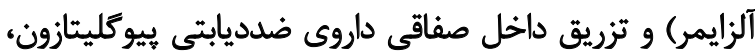

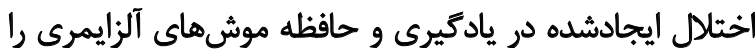

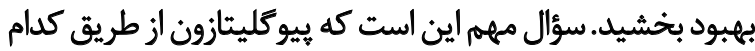
مكانيسم اختلال ياد كيرى و حافظه فضايى موش هائ آلزايمرى را را

$$
\text { بهبود بخشيده است؟ بكاني }
$$

تيازوليدين ديونها احتمالاًاز طريق كاهش مقاومت به انسولين،

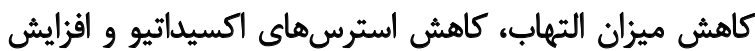
جريان خون مغز مىتوانئد در درمان بيمارى التهاى نورودرثراتيو 


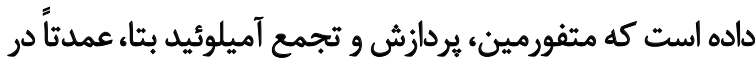

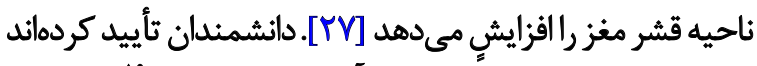

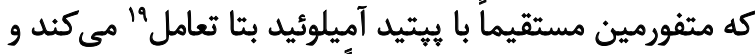

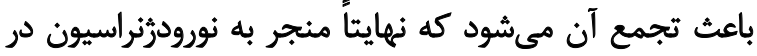

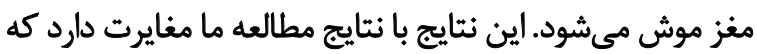

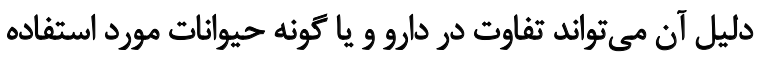

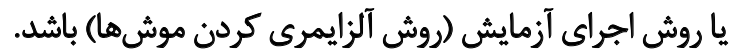

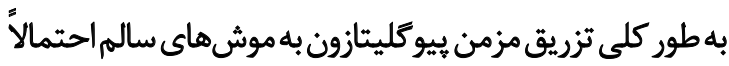

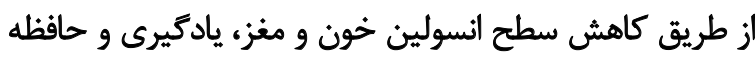

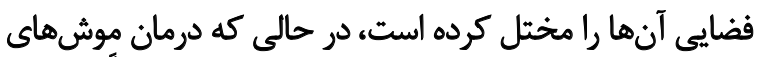

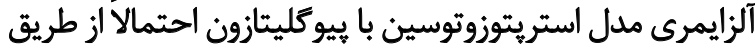

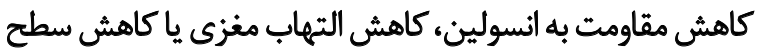

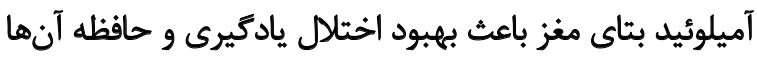

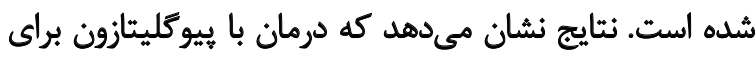
درمان اختلالات شناختى در بيماران آلزايمرى مفيد است.

ملاحظات اغخاقي

$$
\text { يبيروى از اصول اخلاق يُوهش }
$$

اين مطالعه با كُد اخلاق IR.QUMS.REC.1398.158 در عرد دانشعاه علوميزشكى قزوين ثبت شده اسلاق است

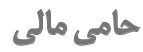

اين مقاله حاصل باياننامه نويسنده سوم، دانشجوى يزشكى

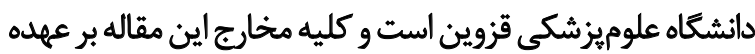

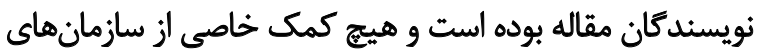

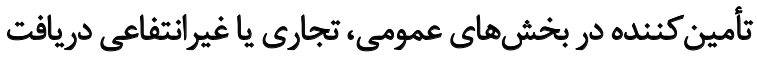

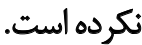

$$
\text { مشاركت ثويسند }
$$

نعارش: همه نويسندكان؛ منابع و اعتبارسنجي: سيده شيما

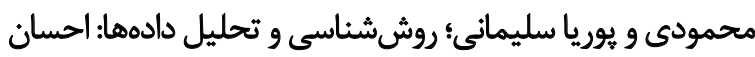

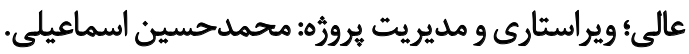

$$
\text { ت تعارض مثناقع }
$$

بنابر اظهار نويسندكان اين مقاله تعارض منافع ندارد.
از داروى يُيوكَليتازون از طريق كاهش استرس اكسيداتيو باعث

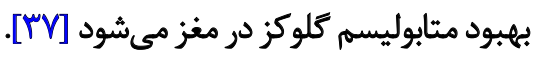

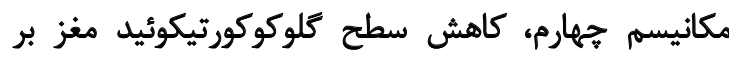

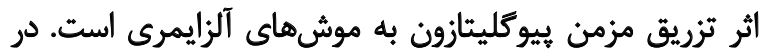

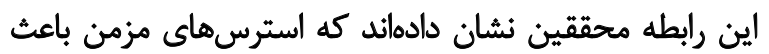

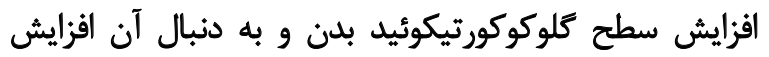

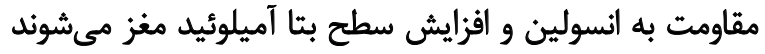

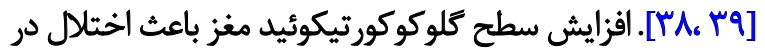

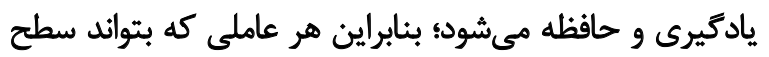

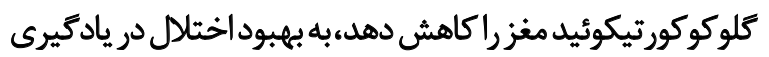

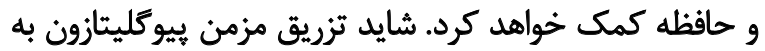

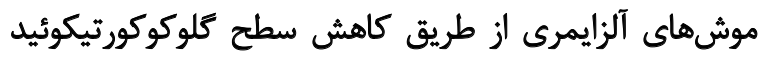

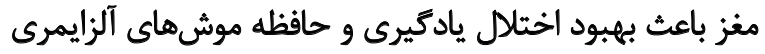

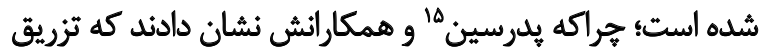

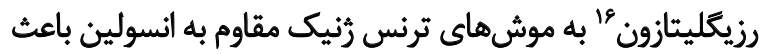

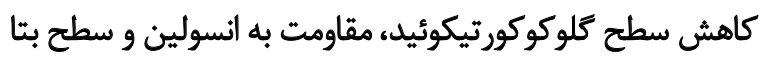

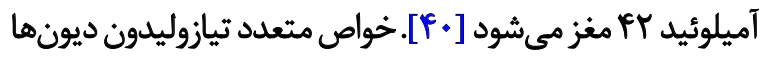

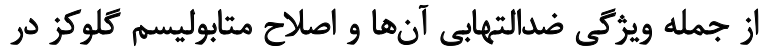

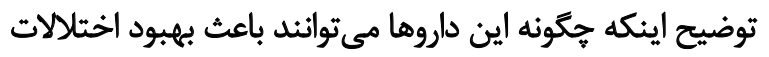

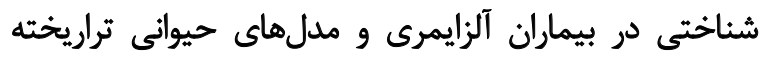

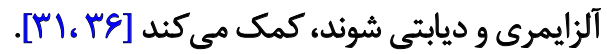

نتايج مرحله دوم ما با نتايج محققينى كه نشان دادهاند

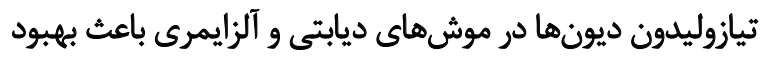

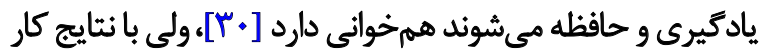

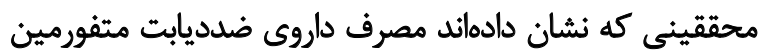

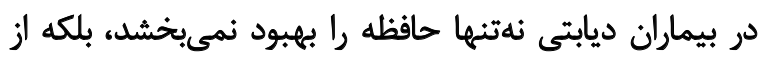

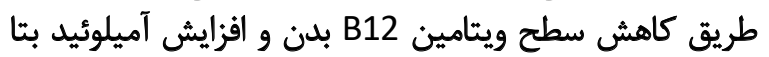

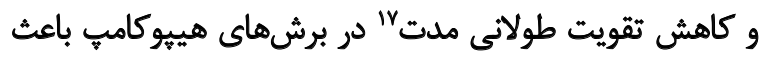

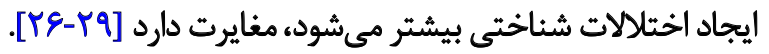

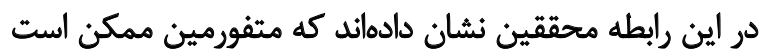

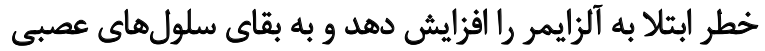

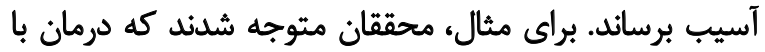

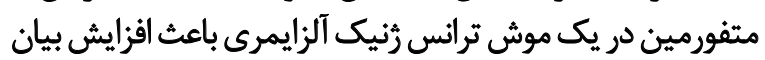

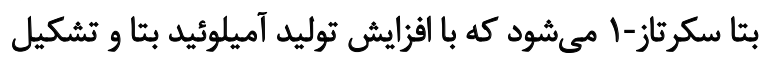

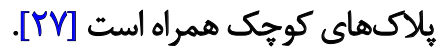

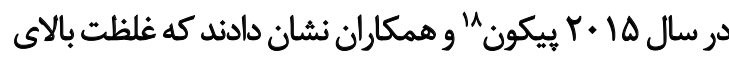

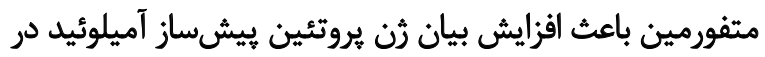

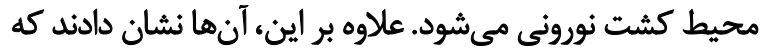

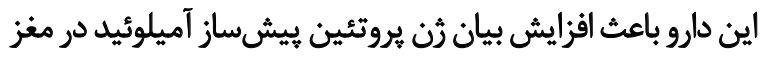

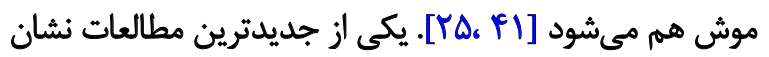

\section{Pedersen}

16. Rosiglitazone

17. Long Term Potentiation (LTP)

18. Picone 


\section{References}

[1] Zhang J, Chen C, Hua Sh, Liao H, Wang M, Xiong Y, et al. An updated meta-analysis of cohort studies: Diabetes and risk of Alzheimer's disease. Diabetes Res Clin Pract. 2017; 124:41-7. [DOI:10.1016/j.diabres.2016.10.024] [PMID]

[2] Biessels GJ, Staekenborg S, Brunner E, Brayne C, Scheltens P. Risk of dementia in diabetes mellitus: A systematic review. Lancet Neurol. 2006; 5(1):64-74. [DOI:10.1016/S14744422(05)70284-2] [PMID]

[3] Accardi G, Caruso C, Colonna-Romano G, Camarda C, Monastero R, Candore G. Can Alzheimer disease be a form of type 3 diabetes? Rejuvenation Res. 2012; 15(2):217-21. [DOI:10.1089/rej.2011.1289] [PMID]

[4] Steen E, Terry BM, Rivera EJ, Cannon JL, Neely TR, Tavares $\mathrm{R}$, et al. Impaired insulin and insulin-like growth factor expression and signaling mechanisms in Alzheimer's disease -- is this type 3 diabetes? J Alzheimers Dis. 2005; 7(1):63-80. [DOI:10.3233/JAD-2005-7107] [PMID]

[5] El-Mir MY, Detaille D, R-Villanueva G, Delgado-Esteban M, Guigas B, Attia $S$, et al. Neuroprotective role of antidiabetic drug metformin against apoptotic cell death in primary cortical neurons. J Mol Neurosci. 2008; 34(1):77-87. [DOI:10.1007/ s12031-007-9002-1] [PMID]

[6] Wang J, Gallagher D, DeVito LM, Cancino GI, Tsui D, He L, et al. Metformin activates an atypical PKC-CBP pathway to promote neurogenesis and enhance spatial memory formation. Cell Stem Cell. 2012; 11(1):23-35. [DOI:10.1016/j. stem.2012.03.016] [PMID]

[7] Gupta A, Bisht B, Dey CS. Peripheral insulin-sensitizer drug metformin ameliorates neuronal insulin resistance and Alzheimer's-like changes. Neuropharmacology. 2011; 60(6):91020. [DOI:10.1016/j.neuropharm.2011.01.033] [PMID]

[8] Zhao WQ, De Felice FG, Fernandez S, Chen H, Lambert MP, Quon MJ, et al. Amyloid beta oligomers induce impairment of neuronal insulin receptors. FASEB J. 2009; 22(1):246-60. [DOI:10.1096/fi.06-7703com] [PMID]

[9] Lester-Coll N, Rivera EJ, Soscia SJ, Doiron K, Wands JR, de la Monte SM. Intracerebral streptozotocin model of type 3 diabetes: Relevance to sporadic Alzheimer's disease. J Alzheimers Dis. 2006; 9(1):13-33. [DOI:10.3233/JAD-2006-9102] [PMID]

[10] Searcy JL, Phelps JT, Pancani T, Kadish I, Popovic J, Anderson $\mathrm{KL}$, et al. Long-term pioglitazone treatment improves learning and attenuates pathological markers in a mouse model of Alzheimer's disease. J Alzheimers Dis. 2012; 30(4):943-61. [DOI:10.3233/JAD-2012-111661] [PMID] [PMCID]

[11] Lamontagne J, Jalbert-Arsenault É, Pepin É, Peyot ML, Ruderman NB, Nolan $\mathrm{CJ}$, et al. Pioglitazone acutely reduces energy metabolism and insulin secretion in rats. Diabetes. 2013; 62(6):2122-9. [DOI:10.2337/db12-0428] [PMID] [PMCID]

[12] Sato T, Hanyu H, Hirao K, Kanetaka H, Sakurai H, Iwamoto T. Efficacy of PPAR- $\gamma$ agonist pioglitazone in mild Alzheimer disease. Neurobiol Aging. 2011; 32(9):1626-33. [DOI:10.1016/j. neurobiolaging.2009.10.009] [PMID]
[13] Esposito K, Ciotola M, Merante D, Giugliano D. Rosiglitazone cools down inflammation in the metabolic syndrome. Arterioscler Thromb Vasc Biol. 2006; 26(6):1413-4. [DOI:10.1161/01. ATV.0000223874.94624.11] [PMID]

[14] Landreth GE, Heneka MT. Anti-inflammatory actions of peroxisome proliferator-activated receptor gamma agonists in Alzheimer's disease. Neurobiol Aging. 2001; 22(6):937-44. [DOI:10.1016/S0197-4580(01)00296-2] [PMID]

[15] Nicolakakis N, Aboulkassim T, Ongali B, Lecrux C, Fernandes $P$, Rosa-Neto $P$, et al. Complete rescue of cerebrovascular function in aged Alzheimer's disease transgenic mice by antioxidants and pioglitazone, a peroxisome proliferator-activated receptor $\gamma$ agonist. J Neurosci. 2008; 28(37):9287-96. [DOI:10.1523/JNEUROSCI.3348-08.2008] [PMID] [PMCID]

[16] To AWM, Ribe EM, Chuang TT, Schroeder JE, Lovestone S. The $\varepsilon 3$ and $\varepsilon 4$ alleles of human APOE differentially affect tau phosphorylation in hyperinsulinemic and pioglitazone treated mice. PLoS One. 2011; 6(2):e16991. [DOI:10.1371/journal. pone.0016991] [PMID] [PMCID]

[17] Pancani T, Phelps JT, Searcy JL, Kilgore MW, Chen KC, Porter NM, et al. Distinct modulation of voltage-gated and ligand-gated $\mathrm{Ca} 2+$ currents by PPAR- $\psi$ agonists in cultured hippocampal neurons. J Neurochem. 2009; 109(6):1800-11. [DOI:10.1111/j.1471-4159.2009.06107.x] [PMID] [PMCID]

[18] Escribano L, Simón AM, Gimeno E, Cuadrado-Tejedor M, López de Maturana R, García-Osta A, et al. Rosiglitazone rescues memory impairment in Alzheimer's transgenic mice: Mechanisms involving a reduced amyloid and tau pathology. Neuropsychopharmacology. 2010; 35(7):1593-604. [DOI:10.1038/npp.2010.32] [PMID] [PMCID]

[19] Yan Q, Zhang J, Liu H, Babu-Khan S, Vassar R, Biere AL, et al. Anti-inflammatory drug therapy alters beta-amyloid processing and deposition in an animal model of Alzheimer's disease. J Neurosci. 2003; 23(20):7504-9. [DOI:10.1523/JNEUROSCI.23-20-07504.2003] [PMID] [PMCID]

[20] Gupta R, Gupta LK. Improvement in long term and visuospatial memory following chronic pioglitazone in mouse model of Alzheimer's disease. Pharmacol Biochem Behav. 2012; 102(2):184-90. [DOI:10.1016/j.pbb.2012.03.028] [PMID]

[21] Esmaeili MH, Enayati M, Khabbaz Abkenar F, Ebrahimian $F$ Salari AA. Glibenclamide mitigates cognitive impairment and hippocampal neuroinflammation in rats with type 2 diabetes and sporadic Alzheimer-like disease. Behav Brain Res. 2020; 379:112359. [DOI:10.1016/j.bbr.2019.112359] [PMID]

[22] Blalock EM, Phelps JT, Pancani T, Searcy JL, Anderson KL, Gant JC, et al. Effects of long-term pioglitazone treatment on peripheral and central markers of aging. PLoS One. 2010; 5(4):e10405. [DOI:10.1371/journal.pone.0010405] [PMID] [PMCID]

[23] de la Monte SM, Tong M, Lester-Coll N, Plater M Jr, Wands $J R$. Therapeutic rescue of neurodegeneration in experimental type 3 diabetes: Relevance to Alzheimer's disease. J Alzheimers Dis. 2006; 10(1):89-109. [DOI:10.3233/JAD-200610113] [PMID] 
[24] Szapary PO, Bloedon LT, Samaha FF, Duffy D, Wolfe ML, Soffer $D$, et al. Effects of pioglitazone on lipoproteins, inflammatory markers, and adipokines in nondiabetic patients with metabolic syndrome. Arterioscler Thromb Vasc Biol. 2006; 26(1):182-8. [DOI:10.1161/01.ATV.0000195790.24531.4f] [PMID]

[25] Picone P, Vilasi S, Librizzi F, Contardi M, Nuzzo D, Caruana L, et al. Biological and biophysics aspects of metformin-induced effects: cortex mitochondrial dysfunction and promotion of toxic amyloid pre-fibrillar aggregates. Aging (Albany NY). 2016; 8(8):1718-34. [DOI:10.18632/aging.101004] [PMID] [PMCID]

[26] Moore EM, Mander AG, Ames D, Kotowicz MA, Carne RP, Brodaty $\mathrm{H}$, et al. Increased risk of cognitive impairment in patients with diabetes is associated with metformin. Diabetes Care. 2013; 36(10):2981-7. [DOI:10.2337/dc13-0229] [PMID] [PMCID]

[27] Chen Y, Zhou K, Wang R, Liu Y, Kwak YD, Ma T, et al. Antidiabetic drug metformin (GlucophageR) increases biogenesis of Alzheimer's amyloid peptides via up-regulating BACE1 transcription. Proc Natl Acad Sci U S A. 2009; 106(10):3907-12. [DOI:10.1073/ pnas.0807991106] [PMID] [PMCID]

[28] Potter WB, O'Riordan KJ, Barnett D, Osting SMK, Wagoner M, Burger $C$, et al. Metabolic regulation of neuronal plasticity by the energy sensor AMPK. PLoS One. 2010; 5(2):e8996. [DOI:10.1371/ journal.pone.0008996] [PMID] [PMCID]

[29] Imfeld P, Bodmer M, Jick SS, Meier CR. Metformin, other antidiabetic drugs, and risk of Alzheimer's disease: A populationbased case-control study. J Am Geriatr Soc. 2012; 60(5):916-21. [DOI:10.1111/j.1532-5415.2012.03916.x] [PMID]

[30] Liu LP, Yan TH, Jiang LY, Hu W, Hu M, Wang C, et al. Pioglitazone ameliorates memory deficits in streptozotocin-induced diabetic mice by reducing brain $\beta$-amyloid through PPAR $\gamma$ activation. Acta Pharmacol Sin. 2013; 34(4):455-63. [DOI:10.1038/aps.2013.11] [PMID] [PMCID]

[31] Chang KL, Wong LR, Pee HN, Yang Sh, Ho PCL. Reverting metabolic dysfunction in cortex and cerebellum of APP/PS1 mice, a model for Alzheimer's disease by pioglitazone, a Peroxisome Proliferator-Activated Receptor Gamma (PPARY) agonist. Mol Neurobiol. 2019; 56(11):7267-83. [DOI:10.1007/s12035-019-1586-2] [PMID]

[32] Jiang LY, Tang SS, Wang XY, Liu LP, Long Y, Hu M, et al. PPARY agonist pioglitazone reverses memory impairment and biochemical changes in a mouse model of type 2 diabetes mellitus. CNS Neurosci Ther. 2012; 18(8):659-66. [DOI:10.1111/j.17555949.2012.00341.x] [PMID] [PMCID]

[33] Hu SH, Jiang T, Yang SS, Yang Y. Pioglitazone ameliorates intracerebral insulin resistance and tau-protein hyperphosphorylation in rats with type 2 diabetes. Exp Clin Endocrinol Diabetes. 2013; 121(4):220-4. [DOI:10.1055/s-0032-1333277] [PMID]

[34] Fernandez-Martos CM, Atkinson RA, Chuah MI, King AE, Vickers JC. Combination treatment with leptin and pioglitazone in a mouse model of Alzheimer's disease. Alzheimers Dement ( $\mathrm{N}$ Y). 2016; 3(1):92-106. [DOI:10.1016/j.trci.2016.11.002] [PMID] [PMCID]

[35] Yang S, Chen Z, Cao M, Li R, Wang Z, Zhang M. Pioglitazone ameliorates $A \beta 42$ deposition in rats with diet-induced insulin resistance associated with AKT/GSK3 $\beta$ activation. Mol Med Rep. 2017; 15(5):2588-94. [DOI:10.3892/mmr.2017.6342] [PMID] [PMCID]
[36] Beheshti F, Hosseini M, Hashemzehi M, Soukhtanloo M, Khazaei $M$, Shafei MN. The effects of PPAR- $\gamma$ agonist pioglitazone on hippocampal cytokines, brain-derived neurotrophic factor, memory impairment, and oxidative stress status in lipopolysaccharide-treated rats. Iran J Basic Med Sci. 2019; 22(8):940-8. [DOI:10.22038/ijbms.2019.36165.8616] [PMID] [PMCID]

[37] Pathan AR, Viswanad B, Sonkusare SK, Ramarao P. Chronic administration of pioglitazone attenuates intracerebroventricular streptozotocin induced-memory impairment in rats. Life Sci. 2006; 79(23):2209-16. [DOI:10.1016/j.lfs.2006.07.018] [PMID]

[38] Mohammad Rezaei R, Pourali-Malabad R, Shiravi A, RashidyPour A, Vafaei AA. Interaction between 5 -HT6 receptors and acute stress and corticosterone on fear memory reconsolidation in mice. Koomesh. 2020; 22(1):185-91. [DOI:10.29252/koomesh.22.1.185]

[39] Choobdar S, Vafaei AA, Rashidy-Pour A, Sedaghat K. Protective role of vitamin-D on chronic stress induced-learning and memory deficits in rats. Koomesh. 2019; 21(4):708-15. [In Persian] http:// koomeshjournal.semums.ac.ir/article-1-5220-en.htm

[40] Pedersen WA, Flynn ER. Insulin resistance contributes to aberrant stress responses in the Tg2576 mouse model of Alzheimer's disease. Neurobiol Dis. 2004; 17(3):500-6. [DOI:10.1016/j. nbd.2004.08.003] [PMID]

[41] Picone P, Nuzzo D, Caruana L, Messina E, Barea A, Vasto S, et al. Metformin increases APP expression and processing via oxidative stress, mitochondrial dysfunction and NF-KB activation: Use of insulin to attenuate metformin's effects. Biochim Biophys Acta. 2015; 1853(5):1046-59. [DOI:10.1016/j.bbamcr.2015.01.017] [PMID] 